\title{
Identification of RR Lyrae stars in the Tycho-2 catalogue
}

\author{
M. O. Mennessier ${ }^{1}$ and J. Colomé2 \\ 1 Université de Montpellier II and CNRS, G.R.A.A.L., cc072, 34095 Montpellier Cedex 5, France \\ 2 Departament d'Astronomia i Meteorologia, Universitat de Barcelona, Avda. Diagonal 647, 8028 Barcelona, Spain
}

\author{
Received 1 February 2002 / Accepted 30 April 2002
}

\begin{abstract}
The Tycho- 2 catalogue provides astrometric and photometric data for the 2.5 million brightest stars in the sky. Therefore it can provide much larger samples than the Hipparcos samples used to date in statistical studies. The object of this paper is the cross-identification of Tycho- 2 sources and known variable stars of RR Lyrae type. The Tycho- 2 data of crossidentified sources are added into the ASTRID specialized database. The present selection almost doubles the size of the sample of RR Lyrae stars with available proper motions.
\end{abstract}

Key words. stars: variables: RR Lyr - catalogs

\section{Introduction}

The Tycho-2 catalogue (Hog et al. 2000) - hereafter TYC2 contains positions, proper motions and two-colour photometric data for the 2.5 million brightest stars in the sky. The completeness is $90 \%$ to $V=11.5 \mathrm{mag}$ and $95 \%$ to $V=11.0$. The main observational basis of the catalogue is the Tycho star-mapper observations by the Hipparcos satellite. About 130 observations per object provide homogeneous photometry from which mean magnitudes in two passbands close to Johnson $B$ and $V$, $B_{\mathrm{T}}$ and $V_{\mathrm{T}}$, were derived.

Many statistical results based on kinematic and photometric Hipparcos data, particularly luminosity calibrations, have been published (see for instance: Fernley et al. 1998; Popowski \& Gould 1998). The luminosity calibration of RR Lyrae stars is one of the steps in the determination of the Universe distance scale (Luri et al. 1998). Some of these statistical results could be greatly improved by using the TYC2. Indeed TYC2 astrometry and photometry are homogeneous and a larger sample size can compensate for less accurate astrometric data. We present the identification of known RR Lyrae variable stars in the TYC2. Section 2 describes the sample of known RR Lyrae stars. The criteria adopted for the cross-identification are given in Sect. 3. Finally, Sect. 4 describes the sample of RR Lyrae stars identified in the TYC2.

\section{RR Lyrae stars}

There are 6543 galactic RR Lyrae stars known. Most of them (6526) are listed in the GCVS (Durlevich et al. 1996) and

Send offprint requests to: $\mathrm{M}$. O. Mennessier,

e-mail: menes@graal.univ-montp2.fr
NSV (Kazarovets et al. 1998) catalogues, and in the more recent Name-list of Variable Stars (Nos. 67-74) published by Kholopov et al. $(1999,2000)$ in the IAU Information Bulletin of Variable Stars. Among the new variable stars discovered by Hipparcos 17 were identified as RR Lyrae stars (Perryman et al. 1997). All of them are included in the ASTRID database ${ }^{1}$.

The Hipparcos catalogue gives measurements for 202 RR Lyrae stars. All of them are in the TYC2 that indicates the Hipparcos identifiers. Among them, 22 belong to the TYC2suplement 1 and 1 to the TYC2-suplement2; these supplements contain respectively stars without TYC2 data and false or heavily disturbed data. Thus only 179 RR Lyrae stars with an Hipparcos identifier will finally be considered. The properties of these 179 stars will be a guide in checking the criteria adopted for the cross-identification (Sect. 3). Let us remark that two stars (MS Ara = HIP 88402 and TZ Aur = HIP 34743) have no proper motion in TYC2 and so may not be useful for any kinematical applications.

Many RR Lyrae stars in the GCVS and NSV are fainter than the magnitude limit of the TYC2. Thus among the 6341 known RR Lyrae stars that were not observed by Hipparcos, only a few more than 400 can be present in TYC2. They are brighter than $12.5 \mathrm{mag}$ in $V$ and $12.8 \mathrm{mag}$ in $P$ at their maximum luminosity.

\section{Criteria of cross-identification}

\subsection{Positions}

The main criterion is the comparison between the positions of known RR Lyrae stars possibly observed by Tycho and

\footnotetext{
${ }^{1}$ http://astrid.graal . univ-montp2.fr
} 


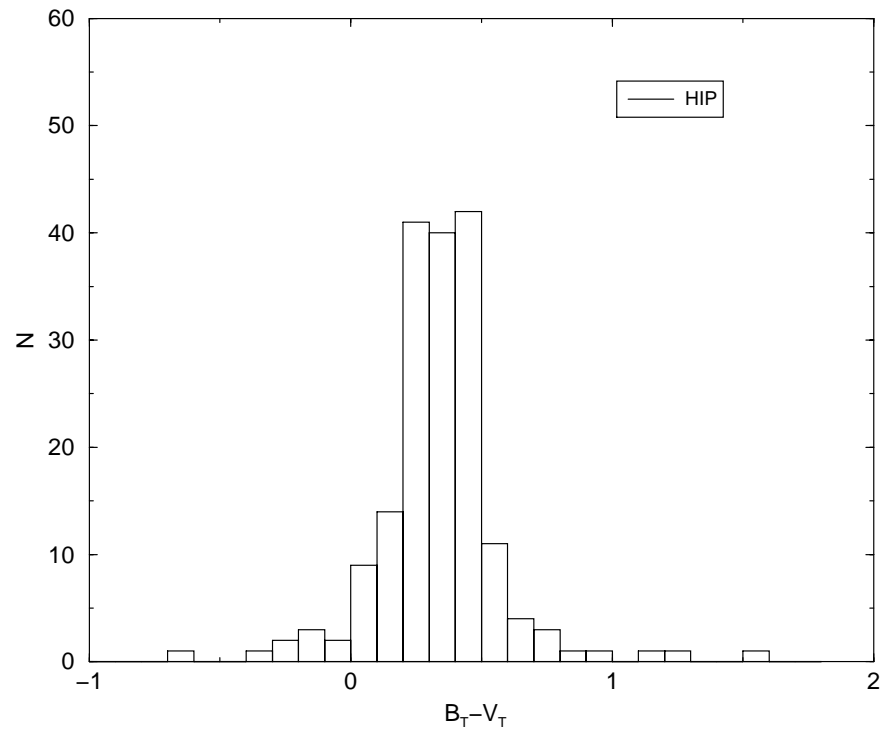

Fig. 1. Histogram of the colour index $\left(B_{\mathrm{T}}-V_{\mathrm{T}}\right)$ for TYC2 RR Lyrae stars with an Hipparcos identifier. $N$ is the number of stars in each 0.1 mag interval.

the positions of TYC2 sources. The epoch of TYC2 positions is J2000.0. Only 345 non-Hipparcos RR Lyrae stars have a J2000.0 position in the SIMBAD database ${ }^{2}$, so we cannot use this database for our purpose, but it allows us to verify the agreement with our J2000.0 GCVS position computation. The comparison of our computed J2000.0 positions and those of SIMBAD for these 345 stars shows that $60 \%$ and $40 \%$ of the differences between the two positions are smaller than $2^{\prime \prime}$ and $1^{\prime \prime}$ respectively, which agrees with the accuracy of the GCVS positions.

As a first step, we retain the TYC2 sources closer than 3 times the mean quadratic error on the TYC2 and GCVS J2000.0 positions of one RR Lyrae star. Thus, 482 TYC2 sources are selected as possibly related to 270 known GCVS non-Hipparcos RR Lyrae stars. In some cases one RR Lyrae star has been matched with more than one TYC 2 source by this first selection. The criteria described in the next sections refine this selection.

\subsection{Colour indices}

It is very important to check that TYC2 sources positionaly matched with a RR Lyrae star have physical characteristics consistent with this type of stars. The $\left(B_{\mathrm{T}}-V_{\mathrm{T}}\right)$ colour should be compatible with the range for RR Lyrae stars, which can be estimated at $[0,0.7]$. The histogram of the observed $\left(B_{\mathrm{T}}-V_{\mathrm{T}}\right)$ for Hipparcos RR Lyrae stars fulfills this assumption, as can be seen in Fig. 1.

Since we are dealing with variable stars the standard error of the mean magnitudes in TYC 2 is large. We denote by $\sigma_{B_{\mathrm{T}}}$ and $\sigma_{V_{\mathrm{T}}}$ the respective accuracy on $B_{\mathrm{T}}$ and $V_{\mathrm{T}}$. The colour criterion adopted for acceptance of a matched TYC2 star is:

$$
\begin{aligned}
& \text { - all sources, if } 0 \leq B_{\mathrm{T}}-V_{\mathrm{T}} \leq 0.7 \\
& \text { - sources with }\left|\left(B_{\mathrm{T}}-V_{\mathrm{T}}\right) /\left(\sigma_{B_{\mathrm{T}}}+\sigma_{V_{\mathrm{T}}}\right)\right| \leq 1 \text {, if } B_{\mathrm{T}}-V_{\mathrm{T}} \leq 0
\end{aligned}
$$

\footnotetext{
${ }^{2}$ http://simbad.u-strasbg.fr/
}

- sources with $\left(\left(B_{\mathrm{T}}-V_{\mathrm{T}}\right)-0.7\right) /\left(\sigma_{B_{\mathrm{T}}}+\sigma_{V_{\mathrm{T}}}\right) \leq 1$, if $B_{\mathrm{T}}-$ $V_{\mathrm{T}} \geq 0.7$.

The error measure $\sigma_{B_{\mathrm{T}}}+\sigma_{V_{\mathrm{T}}}$ is used instead of $\sqrt{\sigma_{B_{\mathrm{T}}}^{2}+\sigma_{V_{\mathrm{T}}}^{2}}$ to have a greater margin of acceptance. This enlarged margin is wide enough to retain faint RR Lyrae stars placed on the galactic plane, in which $\left(B_{\mathrm{T}}-V_{\mathrm{T}}\right)$ is substantially reddened (see Sect. 4). The last two criteria are satisfified by all Hipparcos RR Lyrae stars showing that they are flexible enough to allow good candidates with anomalous colours.

\subsection{Magnitudes}

A second criterion to refine the selection can be defined by taking into account that the TYC2 magnitude of the star must agree with the magnitude range for the matching object in the GCVS. To establish this we define the ratios:

$$
\begin{aligned}
& R_{\max }=\left(P\left(B_{\mathrm{T}}\right)-P_{\max }\right) / \sigma_{B_{\mathrm{T}}} \\
& \text { and } R_{\min }=\left(P_{\min }-P\left(B_{\mathrm{T}}\right)\right) / \sigma_{B_{\mathrm{T}}}
\end{aligned}
$$

or

$R_{\max }=\left(V\left(V_{\mathrm{T}}\right)-V_{\max }\right) / \sigma_{V_{\mathrm{T}}}$

and $R_{\min }=\left(V_{\min }-V\left(V_{\mathrm{T}}\right)\right) / \sigma_{V_{\mathrm{T}}}$

where $P_{\max }$ and $P_{\min }$ (or $V_{\max }$ and $V_{\min }$ ) are respectively, following the GVCS rule, the $P$ (or $V$ ) brightest and faintest magnitudes ever observed for the variable stars ${ }^{3} . P\left(B_{\mathrm{T}}\right)$ and $V\left(V_{\mathrm{T}}\right)$ are the TYC2 magnitudes reduced to photographic and visual magnitudes respectively. There is one pair of values $\left(R_{\max }, R_{\min }\right)$ for each star because there is only one photometric measurement $(P$ or $V)$ in the GCVS. We use the approximate relations derived between Johnson and Tycho systems over the $\left(B_{\mathrm{T}}-V_{\mathrm{T}}\right)$ range of RR Lyrae stars:

$V=V_{\mathrm{T}}-0.09\left(B_{\mathrm{T}}-V_{\mathrm{T}}\right)$

$B=B_{\mathrm{T}}-0.24\left(B_{\mathrm{T}}-V_{\mathrm{T}}\right)$

(Perryman et al. 1997, Vol. 1, Sect. 3), and the unaccurate relation between $B$ and $P$ :

$B=P+0.11$

which is given by Allen (1973) with no indication of the spectral range validity. Applying these approximate corrections could be questionable. However, for the colour range of the RR Lyrae stars, the difference $V-V_{\mathrm{T}}$ is clearly negligible $(-0.02$ on average) and the difference $P-B_{\mathrm{T}}(-0.22$ on average) is smaller or of the same order as the uncertainty on the GCVS magnitudes due to the long-term changes of several tenths of magnitude in the light curves of the RR Lyrae stars (see, for instance, Tsesevich 1969). We have checked that the effect of the correction on the selected stars is marginal.

The distribution of the Hipparcos RR Lyrae stars in the plane $\left(R_{\max }, R_{\min }\right)$ (see Fig. 2, where these ratios are plotted

\footnotetext{
${ }^{3}$ Defining $R_{\max }$ and $R_{\min }$, we have neglected the GCVS errors in extrema magnitudes because they cannot be properly estimated.
} 


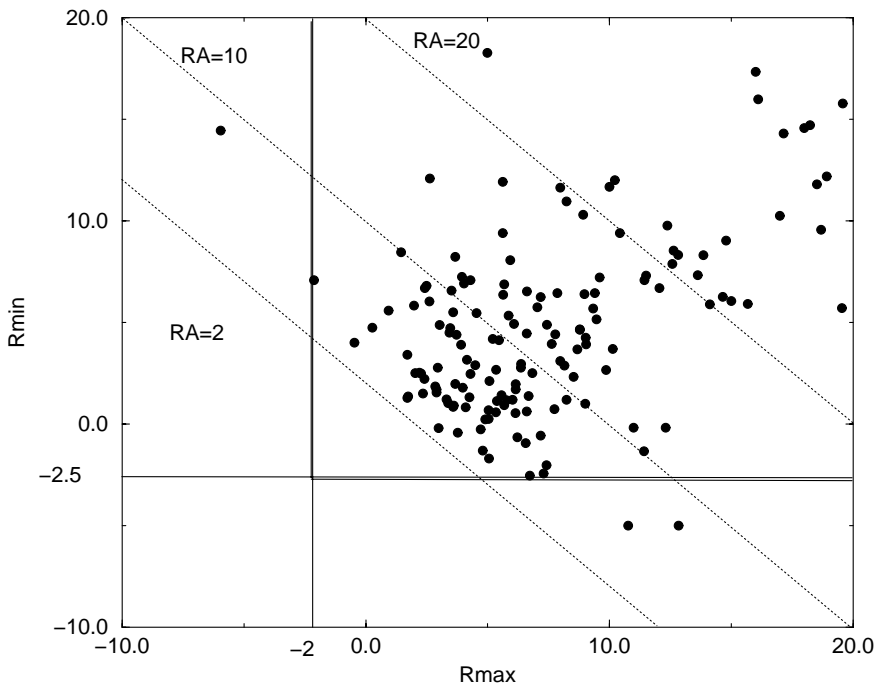

Fig. 2. Distribution of the Hipparcos RR Lyrae stars in the plane $\left(R_{\max }, R_{\min }\right)$.

for all stars simultaneously, i.e. those having photographic and visual magnitudes in the GCVS) helps to determine the acceptance limit for a cross-identification. The mean of $R_{\max }$ is greater than the mean of $R_{\min }$, in agreement with the shapes of the light curves of RR Lyrae stars: a RR Lyrae star spends a larger part of its variability cycle closer to the minimum of luminosity than to the maximum and is thus observed by the satellite more times at fainter luminosities.

Thus, looking at the Hipparcos RR Lyrae star behaviour, we adopt as a criterion for acceptance:

$R_{\max } \geq-2$ and $R_{\min } \geq-2.5$

which can be expressed as:

$P_{\max }-2 \sigma_{B_{\mathrm{T}}} \leq P\left(B_{\mathrm{T}}\right) \leq P_{\min }+2.5 \sigma_{B_{\mathrm{T}}}$

or

$V_{\max }-2 \sigma_{V_{\mathrm{T}}} \leq V\left(V_{\mathrm{T}}\right) \leq V_{\min }+2.5 \sigma_{V_{\mathrm{T}}}$

if the GCVS data are, respectively $P$ or $V$ magnitudes.

These zones are indicated by bold lines in Fig. 2. Five Hipparcos stars are far out of this area (hip 54694, 67227, $78891,101545,112532)$, thus, their cross-identification with a Tycho source as given in the TYC2 catalogue can be doubtful.

\section{Results}

The positions, proper motions, and $B_{\mathrm{T}}, V_{\mathrm{T}}$ magnitudes of TYC2 sources identified as known RR Lyrae stars (Hipparcos and non-Hipparcos) are available in the ASTRID database.

Table 1 shows the number of selected sources after the application of each criteria. At the end of the selection process, 172 TYC2 sources are cross-identified with a non-Hipparcos RR Lyrae star. The physical criteria drastically decrease the number of matches deduced only from the position criterion, showing the importance of taking into account physical properties in a cross-identification procedure.

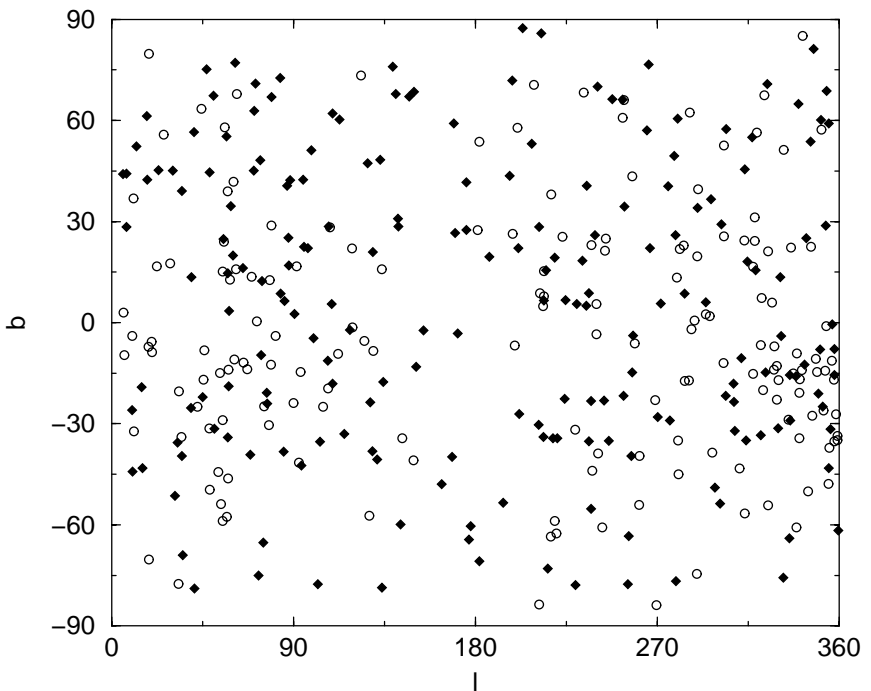

Fig. 3. Distribution of Hipparcos (filled diamonds) and new-identified TYC2 (open circles) RR Lyrae stars in galactic longitude and latitude.

Table 1. Number of known GCVS non-Hipparcos RR Lyrae stars identified with a TYC2 source after each selection step.

\begin{tabular}{lr}
\hline \hline Criterion & Selected sources \\
\hline Position & 270 \\
Colour & $216 / 270$ \\
Magnitude & $172 / 216$ \\
& \\
Total & 172 \\
\hline
\end{tabular}

The list of GCVS and TYC2 identifiers of the 172 nonHipparcos matched RR Lyrae stars is given in Table 4.

Several aspects of the above selection procedure have been checked:

- The selection process does not present zonal effects as a function of the galactic latitude or longitude (see Fig. 3).

- The distribution of non-Hipparcos RR Lyrae stars in the plane $\left(R_{\max }, R_{\min }\right)$ reflects the fact that this kind of variable stars spend a larger part of its variability cycle closer to the minimum of luminosity than to the maximum (see Sect. 3.3), as shown in Fig. 4.

- The variability of TYC2 sources cross-identified with nonHipparcos RR Lyrae stars is checked by using their $\sigma_{V_{\mathrm{T}}}$ values. We compare the $\sigma_{V_{\mathrm{T}}}$ of non-Hipparcos and TYC2 sources to the mean precision of all TYC2 sources within given intervals of $V_{\mathrm{T}}$ magnitude (Hog et al. 2000). Figure 5 shows that the individual $\sigma_{V_{\mathrm{T}}}$ values of nearly all the TYC2 sources cross-identified with a known and non-Hipparcos RR Lyrae star is larger than the TYC2 mean precision. Let us remark that, on average, the $\sigma_{V_{\mathrm{T}}}$ decreases for the faintest stars because of the "survival analysis technique" used to obtain faint Tycho magnitudes (Perryman et al. 1997, Vol. 1, Sect. 3) that is more important for variable 
Table 2. The mean $\sigma_{V_{\mathrm{T}}}$ values within given intervals of $V_{\mathrm{T}}$ magnitude, of the TYC2 sources cross-identified with a known RR Lyrae star and of all TYC2 sources.

\begin{tabular}{lccccc}
\hline \hline Interval of $V_{\mathrm{T}}$ & $8-9$ & $9-10$ & $10-11$ & $11-12$ & $>12$ \\
\hline mean $\sigma_{V_{\mathrm{T}}}$ of RR Lyrae & & & & & \\
Hip stars & 0.015 & 0.025 & 0.053 & 0.118 & 0.199 \\
non-Hip stars & & & & 0.156 & 0.220 \\
\hline mean $\sigma_{V_{\mathrm{T}}}$ of all stars (Hog et al. 2000) & & & & & \\
& 0.014 & 0.023 & 0.050 & 0.114 & 0.198 \\
\hline
\end{tabular}

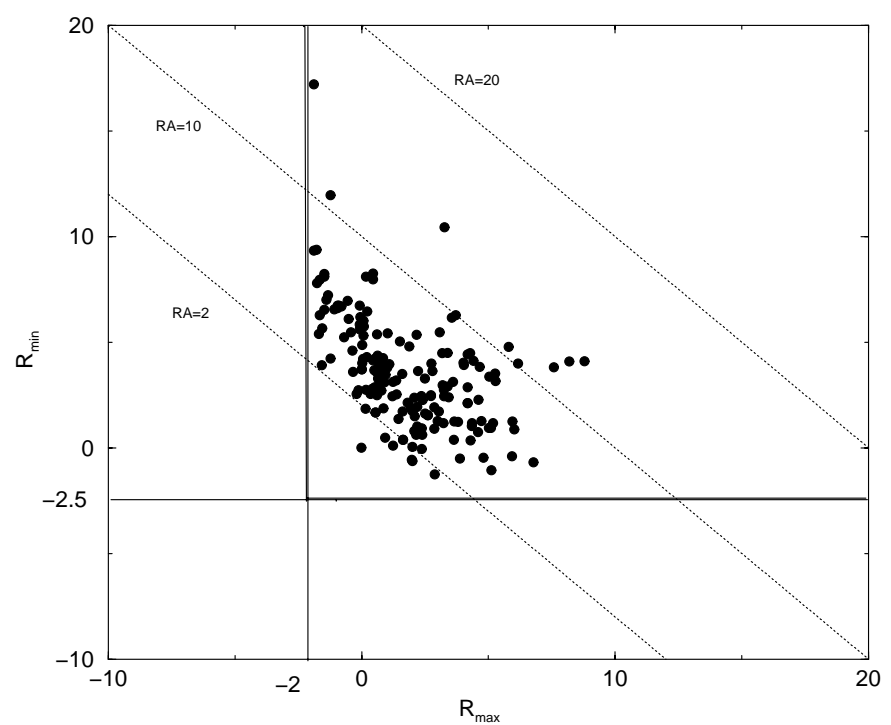

Fig. 4. Distribution of the kept non-Hipparcos RR Lyrae stars in the plane $\left(R_{\max }, R_{\min }\right)$.

stars than for constant stars. There are 4 well-identified stars (SW Boo, IU Cas, BH Pav, ES Peg) with $\sigma_{V_{\mathrm{T}}}$ under the line of mean $\sigma_{V_{\mathrm{T}}}$ values (Fig. 5). However, Table 2 shows that, in all the given intervals of $V_{\mathrm{T}}$ magnitude, the mean $\sigma_{V_{\mathrm{T}}}$ value of the TYC2 sources cross-identified with a known RR Lyrae star is larger than the mean precision of all TYC2 sources, as is to be expected for variable stars. The effect of the censoring is even more important when considering $B_{\mathrm{T}}$ estimates. Thus it is better to use $\sigma_{V_{\mathrm{T}}}$ than $\sigma_{B_{\mathrm{T}}}$ for checking the variability. This check strengthens the classification of these sources as variable stars. Consequently the possibility of a contamination by non-variable stars, if some remains, is only marginal.

- The ratio between the amplitude of the light curve given by the GCVS catalogue and the standard error of the mean TYC2 magnitude:

$R A=\left(P_{\min }-P_{\max }\right) / \sigma_{B_{\mathrm{T}}}$

or

$R A=\left(V_{\min }-V_{\max }\right) / \sigma_{V_{\mathrm{T}}}$

could in principle provide a check for our selection. It is remarkable that the values of $R A$ for the non-Hipparcos RR Lyrae stars are, on average, smaller than those for the

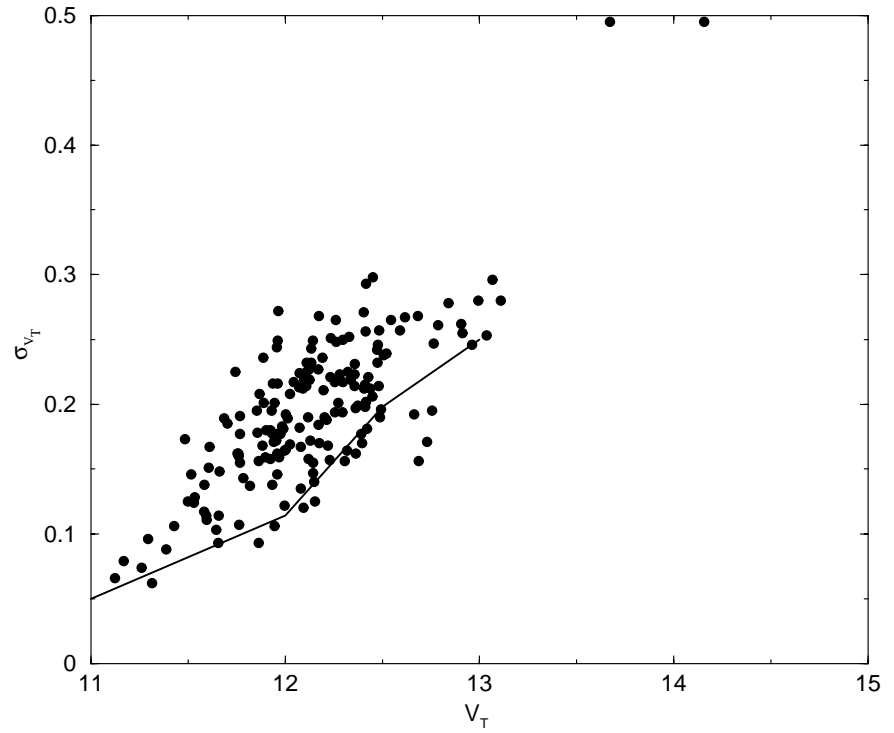

Fig. 5. Comparison of the $\sigma_{V_{\mathrm{T}}}$ of RR Lyrae stars new-identified in TYC2 (open circles) with the mean precision of TYC2 sources within given intervals of $V_{\mathrm{T}}$ magnitude (solid line).

Hipparcos ones because of their fainter magnitudes. Thanks to the large $R A$ values for a great number of sources, one expects that RR Lyrae light curves and periods could be extracted from Tycho multi-epoch photometry. Comparison of periods from Tycho and GCVS should entirely clean the cross-identifications compiled here.

- In a forthcoming paper, Mennessier et al. (2002), we apply the LM method (Luri et al. 1996) to the sample of RR Lyrae stars considered here and make an estimation of the individual distance and, simultaneously, of the interstellar absorption for each star. These results allow us to compute the $\left(B_{\mathrm{T}}-V_{\mathrm{T}}\right)_{0}$ colour indices for Hipparcos and non-Hipparcos RR Lyrae stars, whose histograms are shown in Fig. 6. Both distributions have their maximum at the same colour index range, but there are non-Hipparcos RR Lyrae stars with large intrinsic colour index values. There are two possible reasons for the greater dispersion of the non-Hipparcos RR Lyrae star distribution: these stars are, on average, more distant than the Hipparcos ones and the applied interstellar correction is not accurate enough for them; in addition, they have bigger measurement errors as a result of their fainter magnitudes, as can be seen in Table 3 that shows the median value of $V_{\mathrm{T}}$ and the mean measurement accuracy 

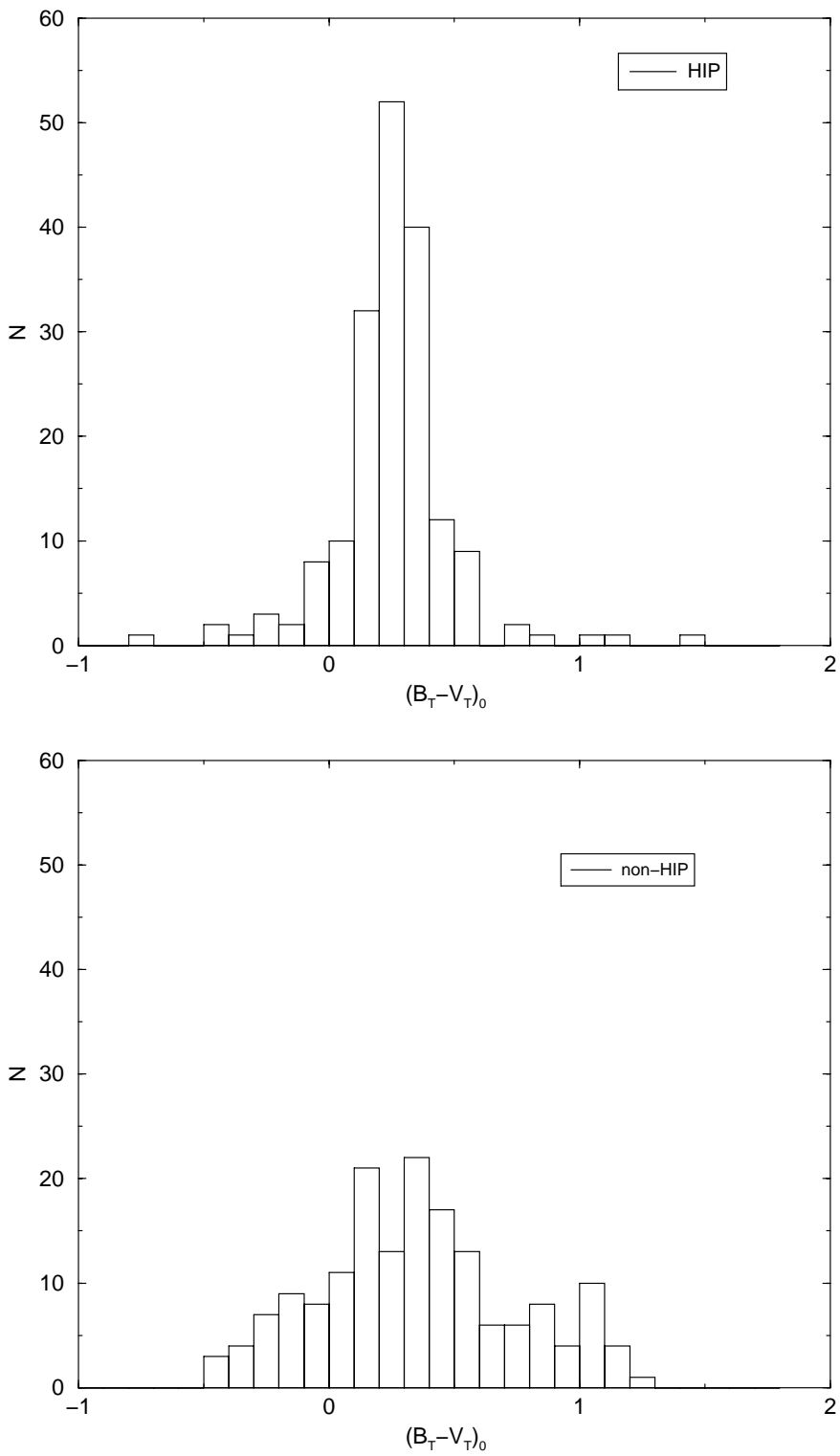

Fig. 6. Histograms of the colour index $\left(B_{\mathrm{T}}-V_{\mathrm{T}}\right)_{0}$ for TYC2 RR Lyrae stars with and without an Hipparcos identifier. $N$ is the number of stars in each 0.1 mag interval.

Table 3. Median values of $V_{\mathrm{T}}$ and mean measurement accuracies of Hipparcos and non-Hipparcos RR Lyrae stars cross-identified with a TYC2 source.

\begin{tabular}{lrr}
\hline \hline & median $V_{\mathrm{T}}$ & $\sigma_{\mathrm{o}}$ \\
\hline non-HIP & 12.13 & 0.32 \\
HIP & 10.98 & 0.08 \\
\hline
\end{tabular}

$\left(\sigma_{\mathrm{o}}=\sqrt{\sigma_{B_{\mathrm{T}}}^{2}+\sigma_{V_{\mathrm{T}}}^{2}}\right)$ for Hipparcos and non-Hipparcos RR Lyrae stars. Both effects will populate the tails and flatten the peak of $\left(B_{\mathrm{T}}-V_{\mathrm{T}}\right)_{0}$ distribution.

\section{Conclusions}

351 TYC2 sources are identified with known RR Lyrae stars: 179 and 172 with and without Hipparcos measurements, respectively.

Among the 179 Hipparcos RR Lyrae stars, 120 have a Tycho identifier in the SIMBAD database.

Only 8 non-Hipparcos GCVS RR Lyrae stars have a Tycho identifier in the SIMBAD database:

- Four of them are not in our list:

- Two of them were considered too faint to have been observed by Tycho: EQ Hya $\left(P_{\max }=12.8\right)$ and V411 $\operatorname{Sgr}\left(P_{\max }=14.0\right)$. The TYC sources associated in SIMBAD have $B_{\mathrm{T}}=11.786$ and 10.706 , respectively, that seems to be a doubtful identification even if we do not consider the difference of photometric systems which is of order of $0.1 \mathrm{mag}$.

- V680 Mon $\left(\left(B_{\mathrm{T}}-V_{\mathrm{T}}\right)=-0.07\right)$ does not pass our colour index criterion, and DR And (11.768 \pm 0.096 for a $P$ magnitude range $12.0-12.8$, respectively) does not pass our magnitude criteria for the cross-identification with TYC2 sources.

- The last three are present in our list of cross-identified sources:

- V674 Cen and AO Tuc agree with our crossidentification,

- V816 Oph, cross-identified with TYC “423 00179 1” differs from our cross-identification. We cross-identify V816 Oph with the Tycho source TYC "423 014931 1". The $V$ magnitude range given by GCVS for V816 Oph is 11.71-13.04. Our TYC2 source has a $V_{\mathrm{T}}$ magnitude value of 12.362 that is in perfect agreement with the GCVS values while TYC "423 001791 " has a $V_{\mathrm{T}}$ magnitude value of 10.967 , out of the $V$ magnitude range of V816 Oph.

The present selection almost doubles the size of the sample of RR Lyrae stars available for statistical studies based on proper motions. Moreover these stars have available $B_{\mathrm{T}}$ and $V_{\mathrm{T}}$ photometry. Their TYC2 identifiers and data have been added into the ASTRID database, where additional astrometric, photometric (visible, infrared, radio) and spectroscopic data can be found. Table 4 gives crossed identifiers for these 172 RR Lyrae stars not included in the Hipparcos catalogue. The TYC2 data are available at the $\mathrm{CDS}^{4}$.

Acknowledgements. This work is supported by the PICASSO program PICS 348, by the Programa Nacional de Astronomía y Astrofísica under contract AYA 2000-0937 and by a Socrates-Erasmus grant. We thank A.Gómez and F.Figueras for fruitful comments, and X.Luri for rereading our manuscript.

\footnotetext{
${ }^{4}$ Catalogue $\mathrm{I} / 259$ is available in electronic form at the CDS via anonymous ftp to cdsarc.u-strasbg.fr (130.79.128.5) or via http://cdsweb.u-strasbg.fr/cgi-bin/qcat?J/A+A/390/173
} 
Table 4. GCVS and TYC2 identifiers of the 172 RR Lyrae non-Hipparcos stars identified with a TYC2 source.

\begin{tabular}{|c|c|c|c|c|c|c|c|}
\hline 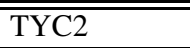 & $\overline{\mathrm{GCVS}}$ & 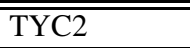 & 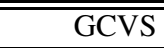 & 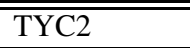 & 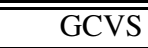 & 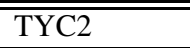 & $\overline{\mathrm{GCVS}}$ \\
\hline 3235006491 & BK And & 7816013001 & V501 Cen & 6027010441 & XX Hya & 6261023611 & V756 Sgr \\
\hline 2773016071 & DM And & 7308014321 & V535 Cen & 5448001071 & DH Hya & 7937015791 & V796 Sgr \\
\hline 9281017421 & BS Aps & 7213019781 & V590 Cen & 6728012361 & FY Hya & 6882003891 & V866 Sgr \\
\hline 9293016371 & LU Aps & 7214015591 & V595 Cen & 0215010361 & GL Hya & 6281018571 & V1025 Sgr \\
\hline 5230005351 & TZ Aqr & 7278003901 & V671 Cen & 5471008171 & IV Hya & 6282007441 & V1055 Sgr \\
\hline 5806001881 & YZ Aqr & 7292010291 & V674 Cen & 9353000881 & SX Hyi & 6278019061 & V1176 Sgr \\
\hline 5814013301 & AA Aqr & 4650009821 & DX Cep & 8444010131 & AO Ind & 7953013621 & V1645 Sgr \\
\hline 5816006161 & BO Aqr & 6482003281 & RT Col & 3204001011 & CQ Lac & 7442015491 & V1646 Sgr \\
\hline 5782004841 & BU Aqr & 1993026581 & RY Com & 1983005391 & RX Leo & 7957004911 & V2277 Sgr \\
\hline 5816002821 & CE Aqr & 1997007541 & BS Com & 0860003681 & AA Leo & 7957011011 & V2281 Sgr \\
\hline 5193007081 & $\mathrm{CP}$ Aqr & 7911010781 & WW CrA & 1437007341 & AE Leo & 6866016811 & V3859 Sgr \\
\hline 5228008281 & FY Aqr & 7916017081 & CV CrA & 0270005191 & AN Leo & 7384004341 & V487 Sco \\
\hline 5181000281 & AA Aql & 7913013561 & V592 CrA & 1976009051 & V LMi & 6782008931 & V765 Sco \\
\hline 5739019861 & KM Aql & 7405014271 & V593 CrA & 3000007441 & X LMi & 6427015151 & RV Scl \\
\hline 1057007881 & V672 Aql & 7906004921 & V629 CrA & 8292014521 & VW Lup & 6985005591 & TX Scl \\
\hline 0500022401 & V793 Aql & 6101000331 & SW Crv & 8303001191 & AW Lup & 7507007151 & UZ Scl \\
\hline 9052015461 & CV Ara & 8973017761 & SW Cru & 7313010841 & GZ Lup & 6999012831 & $\mathrm{AE} \mathrm{Scl}$ \\
\hline 9056007311 & $\mathrm{CZ}$ Ara & 2707007571 & DM Cyg & 2960009581 & RW Lyn & 5705007781 & AF Sct \\
\hline 8746010831 & HX Ara & 3171002851 & V830 Cyg & 2641016781 & RZ Lyr & 0366007111 & AV Ser \\
\hline 8369017661 & QU Ara & 3556020641 & V894 Cyg & 2115020661 & AQ Lyr & 1503005071 & AW Ser \\
\hline 8354007511 & V532 Ara & 3609020521 & V1815 Cyg & 2121021231 & CX Lyr & 1489005101 & DF Ser \\
\hline 1217015081 & RW Ari & 2679013881 & V1823 Cyg & 3120012931 & NR Lyr & 5499008141 & RV Sex \\
\hline 1761019791 & TY Ari & 1632006671 & ZZ Del & 7962010891 & TT Mic & 8744018791 & BI Tel \\
\hline 2559008741 & SW Boo & 1078000341 & CK Del & 0163014821 & V535 Mon & 8762014121 & FU Tel \\
\hline 2569005191 & UU Boo & 1647021351 & EG Del & 9253008981 & ET Mus & 8401001041 & GZ Tel \\
\hline 2022001801 & XX Boo & 1640015181 & FF Del & 8724005411 & VW Nor & 8389014661 & HH Tel \\
\hline 7586002701 & $\mathrm{UCae}$ & 8510016711 & VY Dor & 5080018781 & ST Oph & 9027036281 & TT TrA \\
\hline 4339004741 & UU Cam & 9165007221 & XX Dor & 0423014931 & V816 Oph & 8837004111 & YY Tuc \\
\hline 1927004261 & SS Cnc & 3523014421 & AV Dra & 0717023021 & CM Ori & 8470004931 & AO Tuc \\
\hline 0825006801 & AQ Cnc & 4574016251 & BD Dra & 9058023281 & TY Pav & 9345001321 & BK Tuc \\
\hline 3023009421 & $\mathrm{ZCVn}$ & 3946005311 & CY Dra & 9077020701 & BH Pav & 7671022981 & AN Vel \\
\hline 0168010841 & $\mathrm{X} \mathrm{CMi}$ & 0535012421 & RT Equ & 8785000011 & HV Pav & 7735009751 & FS Vel \\
\hline 0172006421 & RV CMi & 7044015981 & DT Eri & 9084002771 & QR Pav & 0282006321 & UV Vir \\
\hline 0192000431 & AL CMi & 6440007011 & Z For & 1685017841 & VV Peg & 0300003751 & VZ Vir \\
\hline 8627024811 & TX Car & 6440000281 & RS For & 1678003301 & CY Peg & 4965008381 & WW Vir \\
\hline 8957028201 & FT Car & 6442006901 & RX For & 1713013661 & DZ Peg & 4981013291 & AD Vir \\
\hline 8548012141 & IU Car & 7560005261 & SW For & 2735003261 & ES Peg & 0321003061 & AE Vir \\
\hline 3677012131 & HU Cas & 7992003691 & RR Gru & 3671012411 & ET Per & 5538001151 & AS Vir \\
\hline 3650020431 & IU Cas & 8451005531 & AO Gru & 7537000831 & TZ Phe & 0306006471 & BC Vir \\
\hline 4015001501 & NO Cas & 2597005811 & CW Her & 0025004231 & SY Psc & 9198011051 & RV Vol \\
\hline 8642013801 & BI Cen & 3063013331 & GY Her & 6007028201 & AK Pup & 9203015881 & SV Vol \\
\hline 7749015361 & KS Cen & 2608013431 & IP Her & 6542016901 & BQ Pup & 1646001971 & FH Vul \\
\hline 7260003091 & V480 Cen & 8062000841 & UU Hor & 8866014961 & X Ret & 1659006791 & FK Vul \\
\hline
\end{tabular}

\section{References}

Allen, C. W. 1973, Astrophysical Quantities, 3rd ed. (Univ. London, Athlone Press)

Durlevich, O. V., Kasarovets, E. V., Kholopov, P. N., et al. 1996, General Catalogue of Variable Stars, 5th ed. (Moscow State Univ.)

Fernley, J., Barnes, T. G., Skillen, I., et al. 1998, A\&A, 330, 515

Hog, E., Fabricius, C., Makarov, V. V., et al. 2000, The TYCHO2 catalogue, A\&A, 355, L27

Kasarovets, E. V., Durlevich, O. V., \& Samus, N. N. 1998, New Catalogue of Suspected Variable Stars, Inf. Bull. Var. Stars, 4655
Luri, X., Mennessier, M. O., Torra, J., \& Figueras, F. 1996, A\&AS, 117,405

Luri, X., Gomèz, A., Torra, J., et al. 1998, A\&A, 335, 81

Mennessier, M. O., Luri, X., Colome, J., et al. 2002, in preparation

Perryman M., and the HIPPARCOS science team, 1997, ESA, The HIPPARCOS and TYCHO Catalogues, ESA SP-1200

Popowski, P., \& Gould, A. 1998, ApJ, 506, 271

Tsesevich, V. P. 1969, The RR Lyrae stars (Keter Press, Wiener Bindery Ltd, Jerusalem) 